\title{
ON THE REGULARITY OF JOIN-MEET IDEALS OF MODULAR LATTICES
}

\author{
RODICA DINU, VIVIANA ENE, TAKAYUKI HIBI
}

\begin{abstract}
We study join-meet ideals associated with modular non-distributive lattices. We give a lower bound for the regularity and show that they are not linearly related.
\end{abstract}

\section{INTRODUCTION}

Let $L$ be a finite lattice and $K[L]=K\left[x_{a}: a \in L\right]$ be the polynomial ring over the field $K$. The ideal

$$
I_{L}=\left(f_{a b}:=x_{a} x_{b}-x_{a \wedge b} x_{a \vee b}: a, b \in L, a, b \text { incomparable }\right) \subset K[L]
$$

is called the join-meet ideal associated with $L$.

It was proved in [10] that $I_{L}$ is a prime ideal if and only if $L$ is a distributive lattice. In this case, the ring $R[L]=K[L] / I_{L}$ is a normal Cohen-Macaulay domain; see [10]. In literature, the ring $R[L]$ is known as the Hibi ring of the distributive lattice $L$. Hibi rings and the corresponding varieties appear naturally in different combinatorial, algebraic, and geometric contexts; see [3, 4, 10, 11, 14, 15, 20]. Paper [12] surveys the relation between Hibi rings and representation theory.

The starting point of this work was the question whether there exist non-distributive lattices $L$ such that $I_{L}$ has a linear resolution. If $L$ is distributive, by Birkhoff's theorem [1], it follows that $L$ is the lattice of poset ideals $\mathcal{I}(P)$ of the poset $P$ consisting of the join-irreducible elements of $L$. It was proved in [7] that $I_{L}$ has a linear resolution if and only if $L=\mathcal{I}(P)$ where $P$ is the sum of a chain with an isolated element. This result has been recovered in a later paper [5] where it was proved a much stronger result, namely, $\operatorname{reg} I_{L}=|P|-\operatorname{rank} P$. Much less is known about join-meet ideals of non-distributive lattices. As it was observed in [6], if $L$ is modular, but not distributive, the associated join-meet ideal may be not radical.

In this paper we consider join-meet ideals of finite modular lattices. We recall that a finite lattice $L$ is modular if it does not contain any sublattice isomorphic to the pentagon lattice of Figure 1. For basic properties of lattices, like distributivity and modularity, we refer the reader to the monographs [1] and [18].

Every modular lattice $L$ possesses a rank function with the property that

$$
\operatorname{rank} a+\operatorname{rank} b=\operatorname{rank} a \wedge b+\operatorname{rank} a \vee b \text {, for all } a, b \in L \text {. }
$$

If $L$ is a modular lattice, then $L$ is not distributive if and only if it contains a sublattice isomorphic to the diamond lattice of Figure 2.

2010 Mathematics Subject Classification. 05E40, 13H10, $13 \mathrm{D} 02$.

Key words and phrases. Regularity, linear syzygies, lattices, join-meet ideals. 


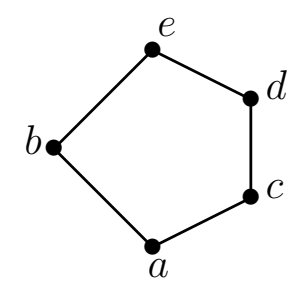

Figure 1. Pentagon lattice

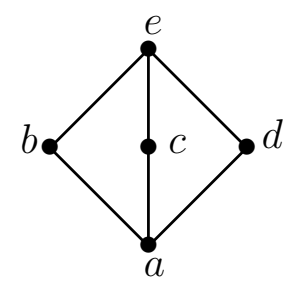

Figure 2. Diamond lattice

By [6, Lemma 1.2], a modular lattice contains a diamond sublattice $L^{\prime}$ such that rank max $L^{\prime}$-rank min $L^{\prime}=2$. This result leads to considering diamond-type lattices with an arbitrary number of elements, say $y_{1}, \ldots, y_{n}$ in between the maximal and the minimal elements. We denote such a lattice by $D_{n+2}$. In Section 1 we study the join-meet ideal of $D_{n+2}$. We show that this ideal is Gorenstein (Theorem 1.6). Moreover, we compute its regularity (Proposition 1.4) and show that it is not linearly related (Proposition 1.8). An equigenerated graded ideal $I$ in a polynomial ring $S$ is called linearly related if it has linear relations.

In Section 2, we show that if $L$ is a finite lattice and $L^{\prime}$ is an induced sublattice of $L$ (see Section 2 for the definition), then $K\left[L^{\prime}\right] / I_{L^{\prime}}$ is an algebra retract of $K[L] / I_{L}$. This leads to the main result of this paper, Theorem $[2.2$, which states that if $L$ is a modular non-distributive lattice, then $\operatorname{reg}\left(K[L] / I_{L}\right) \geq 3$ and, moreover, $I_{L}$ is not linearly related. In particular, the join-meet ideal of a modular non-distributive lattice does not have a linear resolution.

Therefore, combining Theorem [2.2 with [7, Corollary 10] or [5, Corollary 1.2], it follows that if $L$ is a finite modular lattice, then $I_{L}$ has a linear resolution if and only if $L$ is distributive and the poset of join-irreducible elements of $L$ is the sum of a chain with an isolated element.

In addition, Theorem 2.2 shows that, for a modular lattice $L$, if $I_{L}$ is linearly related, then $L$ must be distributive. The planar distributive lattices with linearly related join-meet ideals are completely classified in [2, Theorem 4.12]. On the other hand, there are non-planar distributive lattices whose join-meet ideals are linearly related. For example, if $L=B_{n}$ is the Boolean lattice of rank $n$, it is easily seen that the associated Hibi ring $R[L]=K[L] / I_{L}$ is in fact the Segre product of $n$ polynomials rings in two variables. In [17, Theorem 6$]$ it was shown that the defining ideal of this 
Segre product is linearly related. Consequently, $I_{L}$ is linearly related for $L=B_{n}$, $n \geq 2$.

However, classifying all the distributive lattices whose join-meet ideal is linearly related remains open.

\section{ACKNOWLEDGEMENT}

We thank Mateusz Michałek for drawing our attention to the paper [17] and Florin Ambro for valuable discussions. We gratefully acknowledge the use of the Singular software [8] for computer experiments.

\section{Diamond lattice $D_{n+2}$}

Let us consider the diamond lattice $D_{n+2}, n \geq 3$, with elements $x>y_{1}, \ldots, y_{n}>z$. In Figure 3, we displayed the diamond lattice $D_{7}$.

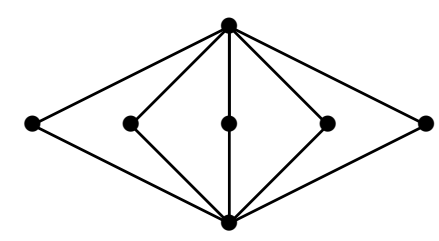

Figure 3. Diamond lattice $D_{7}$

Let $K$ be a field. The join-meet ideal associated to the diamond lattice is

$$
I_{D_{n+2}}=\left(y_{i} y_{j}-x z: 1 \leq i<j \leq n\right) \subseteq K\left[x, y_{1}, \ldots, y_{n}, z\right] .
$$

We denote it simply by $I$ and, also, we denote $S=K\left[x, y_{1}, \ldots, y_{n}, z\right]$ the ring of polynomials in $n+2$ indeterminates over $K$. We consider the following order of the variables $x>y_{1}>\cdots>y_{n}>z$.

We set $f_{i j}:=y_{i} y_{j}-x z$ for $1 \leq i<j \leq n, g_{i}:=x z\left(y_{i}-y_{n}\right)$ for $1 \leq i \leq n-1$, and $h:=x z\left(y_{n}^{2}-x z\right)$.

Proposition 1.1. The reduced Gröbner basis of $I$ with respect to the reverse lexicographic order induced by $x>y_{1}>y_{2}>\cdots>y_{n}>z$ is

$$
\mathcal{G}=\left\{f_{i j}, g_{k}, h: 1 \leq i<j \leq n, 1 \leq k \leq n-1\right\} .
$$

Proof. We apply the Buchberger's Algorithm. Obviously, $\mathcal{G}$ generates $I$. Straightforward calculation shows that all the $S$-polynomials reduce to 0 with respect to $\mathcal{G}$, thus $\mathcal{G}$ is a Gröbner basis of $I$. Moreover, $\mathcal{G}$ is obviously reduced.

Corollary 1.2. The initial ideal of I with respect to the reverse lexicographic order is

$$
\operatorname{in}_{<}(I)=\left(y_{i} y_{j}, x y_{k} z, x y_{n}^{2} z: 1 \leq i<j \leq n, 1 \leq k \leq n-1\right) .
$$

Proposition 1.3. The Hilbert series of $S / I$ and $S / \operatorname{in}_{<}(I)$ is

$$
\operatorname{Hilb}_{S / I}(t)=\operatorname{Hilb}_{S / \mathrm{in}_{<}(I)}=\frac{1+n t+n t^{2}+t^{3}}{(1-t)^{2}} \text {. }
$$


Proof. It is well known that $S / I$ and $S / \operatorname{in}_{<}(I)$ have the same Hilbert series; see, for example, [9, Corollary 6.1.5]. In order to compute the Hilbert series of $S / \operatorname{in}_{<}(I)$, we consider the following exact sequence:

$$
0 \rightarrow \frac{S}{\left(\mathrm{in}_{<}(I): x z\right)}(-2) \rightarrow \frac{S}{\mathrm{in}_{<}(I)} \rightarrow \frac{S}{\left(\mathrm{in}_{<}(I), x z\right)} \rightarrow 0,
$$

where the first non-zero map is the multiplication by $x z$. Then

$$
\operatorname{Hilb}_{\frac{S}{\operatorname{in}_{<}(I)}}(t)=t^{2} \operatorname{Hilb}_{\frac{S}{\left(\mathrm{in}_{<}(I): x z\right)}}(t)+\mathrm{Hilb}_{\frac{S}{\left(\mathrm{in}_{<}(I), x z\right)}}(t) .
$$

Since $S /\left(\operatorname{in}_{<}(I): x z\right)=S /\left(y_{1}, y_{2}, \ldots, y_{n-1}, y_{n}^{2}\right)$ and $y_{1}, y_{2}, \ldots, y_{n-1}, y_{n}^{2}$ is a regular sequence in $S$, it follows that

$$
\operatorname{Hilb}_{\frac{S}{\left(\mathrm{in}_{<}(I): x z\right)}}(t)=\frac{(1-t)^{n-1}\left(1-t^{2}\right)}{(1-t)^{n+2}}=\frac{1+t}{(1-t)^{2}} .
$$

On the other hand,

$$
\frac{S}{\left(\operatorname{in}_{<}(I), x z\right)}=\frac{K\left[y_{1}, \ldots, y_{n}\right]}{\left(y_{i} y_{j}: 1 \leq i<j \leq n\right)} \otimes_{K} \frac{K[x, z]}{(x z)},
$$

which implies that

$$
\operatorname{Hilb}_{\frac{S}{(\operatorname{in}<(I), x z)}}(t)=\left(1+n \frac{t}{1-t}\right) \frac{1+t}{1-t}=\frac{1+n t+(n-1) t^{2}}{(1-t)^{2}}
$$

Therefore,

$$
\operatorname{Hilb}_{\frac{S}{\operatorname{in}_{<}(I)}}(t)=t^{2} \frac{1+t}{(1-t)^{2}}+\frac{1+n t+(n-1) t^{2}}{(1-t)^{2}}=\frac{1+n t+n t^{2}+t^{3}}{(1-t)^{2}} .
$$

Proposition 1.4. (i) The ideals $I$ and $\mathrm{in}_{<}(I)$ are Cohen-Macaulay of dimension 2.

(ii) We have $\operatorname{reg}(S / I)=\operatorname{reg}\left(S / \operatorname{in}_{<}(I)\right)=3$.

(iii) We have $\beta_{n, n+3}(S / I)=\beta_{n, n+3}\left(S / \operatorname{in}_{<}(I)\right)=1$.

Proof. (i). The formula of the Hilbert series of $S / I$ from Proposition 1.3 shows that $\operatorname{dim}(S / I)=\operatorname{dim}\left(S / \operatorname{in}_{<}(I)\right)=2$. By [9, Corollary 3.3.5], it is enough to show that $S /$ in $_{<}(I)$ is Cohen-Macaulay. But this follows easily from the exact sequence (11) since the ends are Cohen-Macaulay of dimension 2, therefore the middle term is also Cohen-Macaulay. Indeed, the left end in (11) is even a complete intersection, while the right end is the tensor product from (2) where the second factor is obviously Cohen-Macaulay and the first factor is the Stanley-Reisner ring of the simplicial complex whose facets are $\left\{y_{1}\right\}, \ldots,\left\{y_{n}\right\}$ which is Cohen-Macaulay.

(ii). Standard arguments for Cohen-Macaulay algebras show that $\operatorname{reg}(S / I)=$ $\operatorname{reg}\left(S / \operatorname{in}_{<}(I)\right)=3$ since the degree of the numerator of the Hilbert series of $S / I$ is equal to 3 ; for more explanation on how to compute the regularity of a standard graded algebra see, for example, [2, Subsection 3.1].

(iii). The highest shift in the resolutions of $S / I$ and $S / \mathrm{in}_{<}(I)$ appears in homological degree $n$ and $\beta_{n, n+3}(S / I)=\beta_{n, n+3}\left(S /\right.$ in $\left._{<}(I)\right)=1$ since the leading coefficient 
in the numerator of the Hilbert series is equal to 1; see the discussion in [4, Section $1]$.

Remark 1.5. One may easily observe that in in $_{<}(I)$ has linear quotients if we order its minimal generators as follows:

$$
y_{1} y_{2}, y_{1} y_{3}, \ldots, y_{1} y_{n}, y_{2} y_{3}, \ldots, y_{2} y_{n}, \ldots, y_{n-1} y_{n}, x y_{1} z, x y_{2} z, \ldots, x y_{n-1} z, x y_{n}^{2} z \text {. }
$$

By using [9, Exercise 8.8], one may compute all the graded Betti numbers of $S / \operatorname{in}_{<}(I)$.

In what follows, we will exploit only that $\beta_{n, n+1}\left(S / \mathrm{in}_{<}(I)\right)=0$. Indeed, by $[9$, Exercise 8.8], we have

$$
\beta_{i+1, i+j}\left(S / \operatorname{in}_{<}(I)\right)=\sum_{k, \operatorname{deg} f_{k}=j}\left(\begin{array}{c}
r_{k} \\
i
\end{array}\right)
$$

where $f_{1}, \ldots, f_{m}$ are the generators of $\operatorname{in}_{<}(I)$ with $m=n(n+1) / 2$ and, for each $k, r_{k}$ denotes the number of variables which generate the ideal quotient $\left(f_{1}, \ldots, f_{k-1}\right): f_{k}$. Then, for $i=n-1$ and $j=2$, we get

$$
\beta_{n, n+1}\left(S / \operatorname{in}_{<}(I)\right)=\sum_{k, \operatorname{deg} f_{k}=2}\left(\begin{array}{c}
r_{k} \\
n-1
\end{array}\right) .
$$

One easily sees that, for every $k$ with $\operatorname{deg} f_{k}=2$, the ideal quotient $\left(f_{1}, \ldots, f_{k-1}\right): f_{k}$ is generated by at most $n-2$ variables, therefore $\beta_{n, n+1}\left(S / \operatorname{in}_{<}(I)\right)=0$.

Note that, by using similar arguments, we get

$$
\beta_{n, n+2}\left(S / \operatorname{in}_{<}(I)\right)=\sum_{k, \operatorname{deg} f_{k}=3}\left(\begin{array}{c}
r_{k} \\
n-1
\end{array}\right)=\sum_{k, \operatorname{deg} f_{k}=3}\left(\begin{array}{l}
n-1 \\
n-1
\end{array}\right)=n-1 .
$$

Theorem 1.6. The ideal I is Gorenstein.

Proof. In order to prove that $\frac{S}{I}$ is a Gorenstein ring, we only need to show that $\beta_{n, n+1}(S / I)=\beta_{n, n+2}(S / I)=0$, since, by Proposition 1.4, we already know that $\beta_{n, n+3}(S / I)=1$. By Remark 1.5, we have $\beta_{n, n+1}\left(S / \operatorname{in}_{<}(I)\right)=0$. Since $\beta_{n, n+1}(S / I) \leq$ $\beta_{n, n+1}\left(S /\right.$ in $\left._{<}(I)\right)$, we get the desired conclusion for $\beta_{n, n+1}(S / I)$.

Therefore, it remains to show that $\beta_{n, n+2}(S / I)=0$.

Let $J=I: z$. Let us consider the following exact sequence of graded $S$-modules:

$$
0 \rightarrow \frac{S}{J}(-1) \stackrel{z}{\rightarrow} \frac{S}{I} \rightarrow \frac{S}{(I, z)} \rightarrow 0 .
$$

We know that $S / I$ is Cohen-Macaulay of $\operatorname{dim}(S / I)=2$ by Proposition 1.4. On the other hand, $S /(I, z) \cong K\left[x, y_{1}, \ldots, y_{n}\right] /\left(y_{i} y_{j}: 1 \leq i<j \leq n\right)$. The ring $K\left[y_{1}, \ldots, y_{n}\right] /\left(y_{i} y_{j}: 1 \leq i<j \leq n\right)$ is the Stanley-Reisner ring of the simplicial complex whose facets are $\left\{y_{1}\right\}, \ldots,\left\{y_{n}\right\}$ which is Cohen-Macaulay, thus $S /(I, z)$ is also Cohen-Macaulay of

$$
\operatorname{dim}(S /(I, z))=\operatorname{dim} K\left[y_{1}, \ldots, y_{n}\right] /\left(y_{i} y_{j}: 1 \leq i<j \leq n\right)+1=2 .
$$

By applying Depth Lemma in (3), it follows that $S / J$ is Cohen-Macaulay of $\operatorname{dim}(S / J)=2$, thus proj $\operatorname{dim}(S / I)=\operatorname{proj} \operatorname{dim}(S / J)=n$. 
From the exact sequence (3), we get the following exact Tor-sequence:

$$
\operatorname{Tor}_{n}\left(\frac{S}{J}(-1), K\right)_{n+2} \rightarrow \operatorname{Tor}_{n}\left(\frac{S}{I}, K\right)_{n+2} \rightarrow \operatorname{Tor}_{n}\left(\frac{S}{(I, z)}, K\right)_{n+2} .
$$

Since $S /(I, z) \cong K\left[x, y_{1}, \ldots, y_{n}\right] /\left(y_{i} y_{j}: 1 \leq i<j \leq n\right)$ and the ideal $\left(y_{i} y_{j}: 1 \leq\right.$ $i<j \leq n$ ) has linear quotients, thus it has a linear resolution, it follows that $\operatorname{Tor}_{n}\left(\frac{S}{(I, z)}, K\right)_{n+2}=0$. We have $\operatorname{Tor}_{n}\left(\frac{S}{J}(-1), K\right)_{n+2}=\operatorname{Tor}_{n}\left(\frac{S}{J}, K\right)_{n+1}$. Therefore, in order to get the desired vanishing of $\beta_{n, n+2}(S / I)$ it is enough to show that $\operatorname{Tor}_{n}\left(\frac{S}{J}, K\right)_{n+1}=0$.

By using [19, Lemma 12.1], a Gröbner basis for $J$ with respect to reverse lexicographic order induced by $x>y_{1}>\ldots>y_{n}>z$, is given by

$$
\left\{y_{i} y_{j}-z x, x\left(y_{k}-y_{n}\right), x\left(y_{n}^{2}-x z\right): 1 \leq i<j \leq n, 1 \leq k \leq n-1\right\} .
$$

If we change the order of variables to $z>y_{1}>\ldots>y_{n}>x$, the Gröbner basis for $J$ does not change. We can consider the following exact sequence:

$$
0 \rightarrow \frac{S}{J: x}(-1) \stackrel{x}{\rightarrow} \frac{S}{J} \rightarrow \frac{S}{(J, x)} \rightarrow 0 .
$$

From (44), we get the exact sequence

$$
\operatorname{Tor}_{n}\left(\frac{S}{J: x}(-1), K\right)_{n+1} \rightarrow \operatorname{Tor}_{n}\left(\frac{S}{J}, K\right)_{n+1} \rightarrow \operatorname{Tor}_{n}\left(\frac{S}{(J, x)}, K\right)_{n+1} .
$$

Note that $\operatorname{Tor}_{n}\left(\frac{S}{J: x}(-1), K\right)_{n+1}=\operatorname{Tor}_{n}\left(\frac{S}{J: x}, K\right)_{n}=0$. Indeed, by using again [19, Lemma 12.1], we derive that $J: x$ is generated by the regular sequence $y_{1}-y_{n}, y_{2}-$ $y_{n}, \ldots, y_{n-1}-y_{n}, y_{n}^{2}-x z$, thus the minimal free resolution of $S / J: x$ is provided by the Koszul complex. The last free module in the resolution is generated in degree $n+1$, hence $\operatorname{Tor}_{n}\left(\frac{S}{J: x}, K\right)_{n}=0$.

In addition, $S /(J, x) \cong \stackrel{n}{K}\left[y_{1}, \ldots, y_{n}, z\right]\left(y_{i} y_{j}: 1 \leq i<j \leq n\right)$ is Cohen-Macaulay of dimension 2, thus proj $\operatorname{dim} S /(J, x)=n-1$, so $\operatorname{Tor}_{n}\left(\frac{S}{(J, x)}, K\right)_{n+1}=0$. Therefore, $\operatorname{Tor}_{n}\left(\frac{S}{J}, K\right)_{n+1}=0$ which yields $\beta_{n, n+2}\left(\frac{S}{I}\right)=0$.

Remark 1.7. We have seen in the above theorem that $S / I$ is a Gorenstein algebra. By Proposition 1.3, the degree of the numerator of the Hilbert series is 3 and the initial degree of $I$ is 2 , thus the algebra $S / I$ is a nearly extremal Gorenstein algebra in the sense of [13].

A homogeneous ideal $I$ in a polynomial ring $R$ which is generated in degree $d$ is called linearly related if its syzygy module is generated by linear relations. In other words, $I$ is linearly related if $\beta_{1 j}(I)=\beta_{2 j}(R / I)=0$ if $j \geq d+2$. The next proposition shows that the join-meet ideal of the diamond lattice $D_{n+2}$ is not linearly related for every $n \geq 3$. 
Proposition 1.8. Let $I \subset S=K[L]$ be the join-meet ideal of $D_{n+2}$. Then $\beta_{24}(S / I)>$ 0 .

Proof. We have seen in the proof of Theorem 1.6 that the ideal $I: x z=(I: z): x=$ $J: x$ is generated by the regular sequence $y_{1}-y_{n}, y_{2}-y_{n}, \ldots, y_{n-1}-y_{n}, y_{n}^{2}-x z$. Let us consider the exact sequence

$$
0 \rightarrow \frac{S}{I: x z}(-2) \stackrel{x z}{\rightarrow} \frac{S}{I} \rightarrow \frac{S}{(I, x z)} \rightarrow 0
$$

and assume that $\beta_{24}(S / I)=\operatorname{dim}_{K} \operatorname{Tor}_{2}(S / I, K)_{4}=0$. From the exact sequence (5), we derive that

$$
0 \rightarrow \operatorname{Tor}_{2}\left(\frac{S}{(I, x z)}, K\right)_{4} \rightarrow \operatorname{Tor}_{1}\left(\frac{S}{I: x z}, K\right)_{2} \rightarrow \operatorname{Tor}_{1}\left(\frac{S}{I}, K\right)_{4}=0
$$

is also an exact sequence. Therefore, we get

$$
\operatorname{Tor}_{2}\left(\frac{S}{(I, x z)}, K\right)_{4} \cong \operatorname{Tor}_{1}\left(\frac{S}{I: x z}, K\right)_{2}
$$

which implies that

$\beta_{24}\left(\frac{S}{(I, x z)}\right)=\operatorname{dim}_{K} \operatorname{Tor}_{2}\left(\frac{S}{(I, x z)}, K\right)_{4}=\operatorname{dim}_{K} \operatorname{Tor}_{1}\left(\frac{S}{I: x z}, K\right)_{2}=\beta_{12}\left(\frac{S}{I: x z}\right)=1$.

On the other hand, we have

$$
\frac{S}{(I, x z)} \cong \frac{K\left[y_{1}, \ldots, y_{n}\right]}{\left(y_{i} y_{j}: 1 \leq i<j \leq n\right)} \otimes_{K} \frac{K[x, z]}{(x z)}
$$

which implies that

$$
\beta_{24}\left(\frac{S}{(I, x z)}\right)=\beta_{12}\left(\frac{K\left[y_{1}, \ldots, y_{n}\right]}{\left(y_{i} y_{j}: 1 \leq i<j \leq n\right)}\right)=\left(\begin{array}{l}
n \\
2
\end{array}\right) .
$$

Consequently, $\left(\begin{array}{l}n \\ 2\end{array}\right)=1$, thus, $n=2$, a contradiction.

\section{A LOWER BOUND FOR THE REGULARITy OF THE JOIN-MEET IDEAL OF A MODULAR NON-DISTRIBUTIVE LATTICE}

Let $L$ be a finite lattice and $I_{L} \subset S=K\left[x_{a}: a \in L\right]$ its associated join-meet ideal. A sublattice $L^{\prime}$ of $L$ is called induced if it has the following property: for every $a, b \in L$, if $a \vee b, a \wedge b \in L^{\prime}$, then $a, b \in L^{\prime}$.

Let us recall that, given the graded $K$-algebras $R^{\prime} \subset R, R^{\prime}$ is an algebra retract of $R$ if there exists a surjective homomorphism of graded algebras $\varepsilon: R \rightarrow R^{\prime}$ such that the restriction of $\varepsilon$ to $R^{\prime}$ is the identity of $R^{\prime}$.

Proposition 2.1. Let $L$ be a finite lattice, $L^{\prime}$ an induced sublattice in $L$, and let $I_{L^{\prime}} \subset K\left[x_{a}: a \in L^{\prime}\right]$ be its associated ideal. Then $R^{\prime}=S^{\prime} / I_{L^{\prime}}$ is an algebra retract of $R=S / I_{L}$. 
Proof. First, we show that $I_{L} \cap S^{\prime}=I_{L^{\prime}}$. Obviously, $I_{L^{\prime}} \subset I_{L} \cap S^{\prime}$. Let $f \in I_{L} \cap$ $S^{\prime}$. Then $f=\sum_{a, b}\left(x_{a} x_{b}-x_{a \vee b} x_{a \wedge b}\right) g_{a b}$, where the sum is taken over $a, b \in L$, incomparable, and $g_{a b} \in S$.

We map $x_{a} \mapsto 0$ for all $a \in L \backslash L^{\prime}$. If $a \in L \backslash L^{\prime}$, that is, $x_{a} \mapsto 0$, then $a \vee b \notin L^{\prime}$ or $a \wedge b \notin L^{\prime}$ since $L^{\prime}$ is induced in $L$. Hence, $x_{a \vee b} \mapsto 0$ or $x_{a \wedge b} \mapsto 0$. Conversely, if $a \vee b \notin L^{\prime}$ or $a \wedge b \notin L^{\prime}$, then $a \notin L^{\prime}$ or $b \notin L^{\prime}$ since $L^{\prime}$ is a sublattice in $L$.

Consequently, after mapping $x_{a}$ to 0 for any $a \in L \backslash L^{\prime}$, we get

$$
f=\sum_{b, c}\left(x_{b} x_{c}-x_{b \vee c} x_{b \wedge c}\right) g_{b c}^{\prime}
$$

where the sum is taken over $b, c \in L^{\prime}$ incomparable, and $g_{b c}^{\prime} \in S^{\prime}$, thus $f \in I_{L^{\prime}}$.

As $I_{L} \cap S^{\prime}=I_{L^{\prime}}$, we have an injective $K$-algebra homomorphism $R^{\prime} \rightarrow R$ and the map $\varepsilon: R \rightarrow R^{\prime}$ induced by $x_{a} \mapsto 0$ for $a \in L \backslash L^{\prime}$ is a retraction map.

Theorem 2.2. Let $L$ be a finite modular non-distributive lattice $L$ and $I_{L} \subset S=$ $K[L]$ its join-meet ideal. Then

(i) $\operatorname{reg} S / I_{L} \geq 3$;

(ii) $\beta_{24}\left(S / I_{L}\right)>0$, thus $I_{L}$ is not a linearly related ideal.

In particular, $S / I_{L}$ does not have a linear resolution.

Proof. Since $L$ is a modular non-distributive lattice, by [6, Lemma 1.2], there is a diamond sublattice $L_{1}$ of $L$ such that rank max $L_{1}-\operatorname{rank} \min L_{1}=2$. Let $x=\max L_{1}$ and $z=\min L_{1}$. We consider the induced sublattice $L^{\prime}$ of $L$ with $x=\max L^{\prime}$ and $z=\min L^{\prime}$. Then $L^{\prime}$ is a diamond sublattice of $L$. Let $I_{L^{\prime}} \subset S^{\prime}=K\left[L^{\prime}\right]$ its associated ideal. By Proposition [2.1, we know that $S^{\prime} / I_{L^{\prime}}$ is an algebra retract of $S / I_{L}$. By [16, Corollary 2.5], we have $\beta_{i j}\left(S^{\prime} / I_{L^{\prime}}\right) \leq \beta_{i j}\left(S / I_{L}\right)$ for all $i, j$. In particular, $\operatorname{reg}\left(S^{\prime} / I_{L^{\prime}}\right) \leq \operatorname{reg}\left(S / I_{L}\right)$. By Proposition 1.4, we have $\operatorname{reg}\left(S^{\prime} / I_{L^{\prime}}\right)=3$, thus the statement (i) holds. By using Proposition 1.8, we get $\beta_{24}\left(S / I_{L}\right) \geq \beta_{24}\left(S^{\prime} / I_{L^{\prime}}\right)>0$, thus we have (ii).

Example 2.3. Here there are some examples of regularity 3.
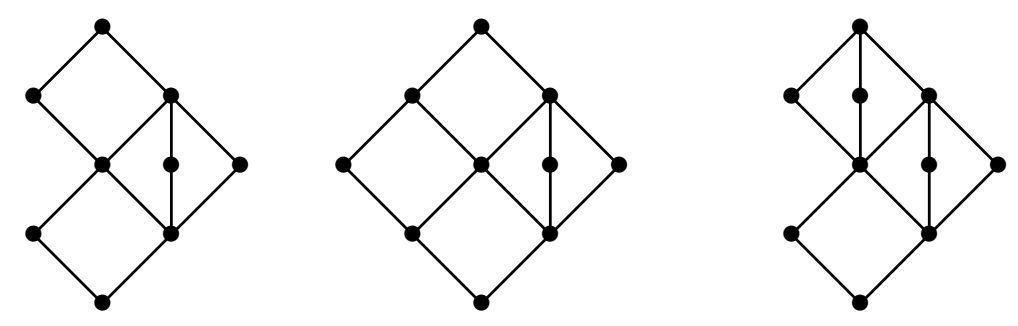

FiguRE 4. Regularity $S / I_{L}=3$

Example 2.4. This example shows two lattices of regularity 5. 

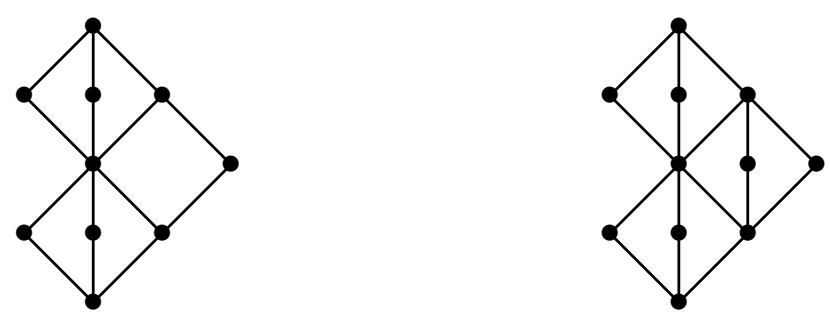

FiguRE 5. Regularity $S / I_{L}=5$
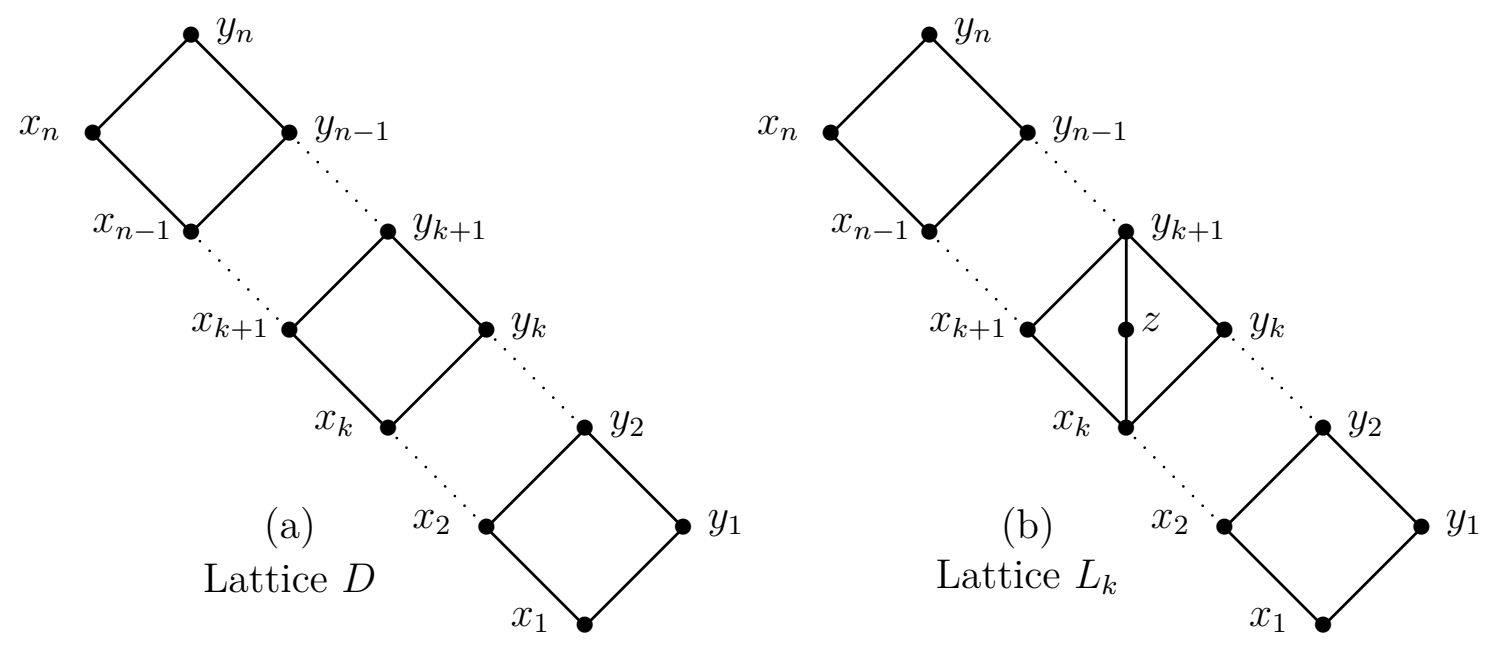

FiguRE 6

Example 2.5. The following class of non-distributive modular lattices was considered in [6].

Let $P$ be the poset which is the sum of a chain and and extra point, and $D=\mathcal{I}(P)$ be the corresponding distributive lattice with the elements labeled as in Figure 6 (a). For every $1 \leq k \leq n-1$, we denote by $L_{k}$ the lattice of Figure 6 (b). Let $I=I_{L_{k}} \subset S=K\left[x_{1}, \ldots, x_{n}, y_{1}, \ldots, y_{n}, z\right]$ be the join-meet ideal of $L_{k}$. We consider the lexicographic order on $S$ induced by $x_{1}>\cdots>x_{n}>y_{1}>\cdots>y_{n}>z$. As it was proved in [6. Lemma 3.2], the initial ideal of $I$ with respect to this order is generated by the following set of monomials:

$$
\begin{gathered}
\left\{x_{j} y_{i}: 1 \leq i<j \leq n\right\} \cup\left\{x_{i} y_{k+1}: 1 \leq i<k\right\} \cup\left\{x_{k} y_{j}: k+1 \leq j \leq n\right\} \\
\cup\left\{x_{i} x_{k+1} y_{j}, x_{i} y_{k} y_{j}: 1 \leq i<k<k+1<j \leq n\right\} \cup\left\{y_{i} y_{k} z: 1 \leq i \leq k\right\} \cup\left\{x_{k+1} z\right\} .
\end{gathered}
$$

Let $J=\operatorname{in}_{<}(I)$. We claim that $\operatorname{reg}(S / J) \leq 3$. Then, $\operatorname{reg}(S / I) \leq 3$. The opposite inequality follows by Theorem 2.2 , thus we get $\operatorname{reg}(S / I)=\operatorname{reg}\left(S / \operatorname{in}_{<}(I)\right)=3$. We now sketch the proof of inequality $\operatorname{reg}(S / J) \leq 3$. We consider the following short 
exact sequences:

$$
0 \rightarrow \frac{S}{J: y_{k+1}}(-1) \stackrel{y_{k+1}}{\rightarrow} \frac{S}{J} \rightarrow \frac{S}{\left(J, y_{k+1}\right)} \rightarrow 0
$$

and

$$
0 \rightarrow \frac{S}{\left(J, y_{k+1}\right): y_{k}}(-1) \stackrel{y_{k}}{\rightarrow} \frac{S}{\left(J, y_{k+1}\right)} \rightarrow \frac{S}{\left(J, y_{k+1}, y_{k}\right)} \rightarrow 0
$$

We have:

$$
\begin{gathered}
J: y_{k+1}=\left(x_{1}, \ldots, x_{k}, x_{k+2}, \ldots, x_{n}\right)+x_{k+1}\left(y_{1}, \ldots, y_{k}, z\right)+\left(y_{1} y_{k} z, \ldots, y_{k-1} y_{k} z, y_{k}^{2} z\right) \\
\left(J, y_{k+1}\right): y_{k}=\left(y_{k+1}, x_{k+1}, x_{k+2}, \ldots, x_{n}\right)+\sum_{j=1}^{k} x_{j}\left(y_{k+2}, \ldots, y_{n}\right)+\sum_{j=2}^{k} x_{j}\left(y_{1}, \ldots, y_{j-1}\right) \\
+\left(y_{1}, \ldots, y_{k}\right) z
\end{gathered}
$$

and

$$
\begin{gathered}
\left(J, y_{k+1}, y_{k}\right)=\left(y_{k+1}, y_{k}\right)+\left(x_{2} y_{1}\right)+x_{3}\left(y_{2}, y_{1}\right)+\cdots+x_{k}\left(y_{1}, \ldots, y_{k-1}\right) \\
+x_{k+1}\left(y_{1}, \ldots, y_{k-1}, z\right)+x_{k+2}\left(y_{1}, \ldots, y_{k-1}\right)+x_{k+3}\left(y_{1}, \ldots, y_{k-1}, y_{k+2}\right)+\cdots \\
+x_{n}\left(y_{1}, \ldots, y_{k-1}, y_{k+2}, \ldots, y_{n}\right)
\end{gathered}
$$

One may easily check that each of these ideals have linear quotients, thus they are componentwise linear. Therefore, we get

$$
\operatorname{reg} \frac{S}{J: y_{k+1}}=2, \operatorname{reg} \frac{S}{\left(J, y_{k+1}\right): y_{k}}=1, \text { and } \operatorname{reg} \frac{S}{\left(J, y_{k+1}, y_{k}\right)}=1 .
$$

From the exact sequence (7), we get $\operatorname{reg} S /\left(J, y_{k+1}\right)=2$, and, replacing in (6) , we derive that $\operatorname{reg} S / J \leq 3$.

The above examples show that there are many lattices of minimal regularity. It would be interesting to find a characterization of the modular non-distributive lattices $L$ for which $\operatorname{reg} S / I_{L}=3$.

\section{REFERENCES}

[1] G. Birkhoff, Lattice Theory (3rd ed.), Amer. Math. Soc. Colloq. Publ. No. 25. Providence, R. I.: Amer. Math. Soc. 1940

[2] V. Ene, Syzygies of Hibi rings, Acta Math. Vietnam., Special Issue on: Commutative Algebra and its Interaction with Algebraic Geometry and Combinatorics II 40(2015) no. 3, 403-446.

[3] V. Ene, J. Herzog, T. Hibi, Linearly related polyominoes, J. Algebraic Combin. 41 (2015), 949-968.

[4] V. Ene, J. Herzog, T. Hibi, S. Saeedi Madani Pseudo-Gorenstein and level Hibi rings, J. Algebra 431 (2015), 138-161.

[5] V. Ene, J. Herzog, S. Saeedi Madani, A note on the regularity of Hibi rings, Manuscripta Math. 148 no. 3-4 (2015), 501-506.

[6] V. Ene, T. Hibi, The join-meet ideal of a finite lattice, J. Commut. Algebra 5(2) (2013), 209-230.

[7] V. Ene, A. A. Qureshi, A. Rauf, Regularity of join-meet ideals of distributive lattices, Electron. J. Combin. 20 (3) (2013), \#P20.

[8] G.-M. Greuel, G. Pfister and H. Schönemann: Singular 2.0. A Computer Algebra System for Polynomial Computations. Centre for Computer Algebra, University of Kaiserslautern, (2001), http://www.singular.uni-kl.de 
[9] J. Herzog, T. Hibi, Monomial ideals, Grad. Texts in Math. 260, Springer, Berlin, 2010.

[10] T. Hibi, Distributive lattices, affine semigroup rings and algebras with straightening laws, In: "Commutative Algebra and Combinatorics" (M. Nagata and H. Matsumura, Eds.), Adv. Stud. Pure Math. 11, North-Holland, Amsterdam, (1987), 93-109.

[11] R. Howe, Pieri algebras and Hibi algebras in representation theory. Symmetry: representantion theory and its applications, 353-384, Progr. Math. 257, Birkhäuser/Springer, New York, 2014.

[12] S. Kim, V. Protsak, Hibi algebras and representation theory, Acta Math. Vietnam. doi.org/10.1007/s40306-018-0263-2

[13] C. Kumar, P. Singh, A. Kumar, Nearly extremal Cohen-Macaulay and Gorenstein algebras, Bull. Austral. Math. Soc. 75 (2007), 211-220.

[14] V. Lakshmibai, J. Brown, Flag Varieties, Texts Read. Math. 53 Hindustan Book Agency, New Delhi, 2009.

[15] V. Lakshmibai, H. Mukherjee, Singular loci of Hibi toric varieties, J. Ramanujan Math. Soc. 26 (2011), 1-29.

[16] H. Ohsugi, J. Herzog, T. Hibi, Combinatorial pure subrings, Osaka J. Math. 37 (2000), 745757.

[17] E. Rubei, On syzygies of Segre embeddings, Proc. Amer. Math. Soc. 130 (12) (2002), 34833493.

[18] R. P. Stanley, Enumerative Combinatorics, Volume I, Wadsworth \& Brooks/Cole, Monterey, CA, 1986.

[19] B. Sturmfels, Gröbner Bases and Convex Polytopes, Amer. Math. Soc., 1995.

[20] D. G. Wagner, Singularities of toric varieties associated with finite distributive lattices, J. Algebraic Combin. 5 (2) (1996), 149-165.

Rodica Dinu, Faculty of Mathematics and Computer Science, University of Bucharest, Str. Academiei 14, Bucharest - 010014, Romania

E-mail address: rodica.dinu93@yahoo.com

Viviana Ene, Faculty of Mathematics and Computer Science, Ovidius University, Bd. Mamaia 124, 900527 Constanta, Romania

E-mail address: vivian@univ-ovidius.ro

Takayuki Hibi, Department of Pure and Applied Mathematics, Graduate School of Information Science and Technology, Osaka University, Suita, Osaka 565-0871, JAPAN

E-mail address: hibi@math.sci.osaka-u.ac.jp 